\title{
Hyperglycemic and Hyperlipidemic Effect of Some Coca-Cola Soft Drinks in Wistar Rats
}

\author{
Augustine I Airaodion $^{1 *}$, Uloaku Ogbuagu ${ }^{1}$, John A Ekenjoku², Emmanuel 0 Ogbuagu $^{2}$, and Edith 0 Airaodion $^{3}$ \\ ${ }^{1}$ Department of Biochemistry, Federal University of Technology, Owerri, Imo State, Nigeria \\ ${ }^{2}$ Department of Pharmacology and Therapeutics, Abia State University, Uturu, Nigeria \\ ${ }^{3}$ Department of Biochemistry, Ladoke Akintola University of Technology, Ogbomoso, Nigeria \\ *Corresponding Author: Augustine I Airaodion, Department of Biochemistry, Federal University of Technology, Owerri, Imo State, \\ Nigeria.
}

Received: November 04, 2019; Published: November 12, 2019

DOI: 10.31080/ASNH.2019.03.0544

\begin{abstract}
This aim of this study is to investigate the effect of some Coca-Cola soft drinks in fasting blood glucose and lipid profile of Wistar rats. 35 adult Wistar rats were used for this study. They were randomly divided into 5 groups of 5 rats each after 7 days acclimatization. They were treated according: animals in group 1 were administered distilled water, those in group 2 were administered Coke, those in group 3 were administered Zero Coke, those in group 4 were administered Fanta, while those in group 5 were administered Sprite. The administration was done orally at a dose of $3 \mathrm{~mL}$ per $100 \mathrm{~g}$ body weight 12 hourly for fourteen days. At the end of administration period, the animals were fasted overnight and anaesthetized using diethyl ether. Blood samples were collected by cardiac puncture. Fasting blood glucose and lipid profile were determined using standard methods. All the soft drinks used in this study (except zero Coke) significantly increased the fasting blood glucose and lipid profile of animals when compared with those of animals in the control group at $\mathrm{p}<0.05$. However, the effect of Sprite on these parameters was not as severe those of Coke and Fanta. The result of this study implies that Coke and Fanta might be deleterious to health as far as hyperglycemia and hyperlipidemia is concerned. Individuals with diabetic family history should minimize their consumption of these drinks but rather consume Zero Coke.
\end{abstract}

Keywords: Coca-Cola Soft Drinks; Fasting Blood Glucose; Lipid Profile; Diabetes; Cardiovascular Diseases

\section{Introduction}

Carbonated soft drinks are sweetened water-based nonalcoholic beverages mostly with balance acidity [1]. They are also known as ready-to-drink beverages. Soft drinks are frequently flavored and coloured and the principal component being water which is needed for hydration. Soft drinks are commonly consumed by both young and old people [2,3]. A notable finding of Wolff and Dangsinger [4] was that weight gained was more dramatic from soft drinks compared with fruit punches and fruit juice. In addition, intake of fruit juice was not associated with an increased risk of type- 2 diabetes. This could be because of the low glycemic index (GI) of fruit juice, soluble fibre, or other constituents of fruit juice that could be beneficial, as the authors suggested [5].

Previous study revealed that a woman with high intake of sugar-sweetened soft drinks tends to be less physically active; in addition, she has high total calories and low protein, alcohol, magnesium and cereal fiber [4]. Also, intake of total carbohydrate, sucrose and fructose as well as overall glycemic index was high in this woman. In essence this woman has dietary pattern and lifestyle that lead to increased risk of several disease states, including obesity, type -2 diabetes and cardiovascular diseases [6]
Sugar sweetened beverages consumption as a marker of an unhealthy lifestyle has the potential of being a quick screening test for increase of obesity and type- 2 diabetes, but it requires validation [7-10]. Because of the large amount of calories in sugar sweetened soft drinks and the relationship between the consumption of these drinks and weight gained, reducing sugar-sweetened beverage consumption may be the simple opportunity to curb the obesity epidemic [11,12]. Obesity is now a complex worldwide problem, resulting from a combination of genetic, behavioral, cultural and environmental influence that calls for not only behavioral changes at individual levels, but also changes in public policy, social, environmental, and cultural norms $[13,14]$.

The world health organization (WHO) and the food and agriculture organization submitted a report in April, 2003 concluding that many death attributed to chronic diseases are due to obesity and outlined how millions of people around the world can avoid chronic diseases through diet and exercise [15]. In the 1960s, for example, diabetes mellitus was said to be rare in the African continent with a prevalent rate of $0.5 \%$ and the prevalent rate, then, in south Africa and north Africa being the highest, but in 1992, Nigeria has a prevalent rate of $2.8 \%$ as discovered by the Nigerian National Expert Committee on non-communicable diseases with more prevalence 
in Urban compared to rural areas [16]. This is as a result of gradual westernization, leading to increase in the number of soft-drinks manufacturing companies. The traditional habit of giving water to a visitor is now replaced by soft drinks or alcohol, hence the need to assess their effects in raising the blood glucose concentration, and lipid profile being the major factors associated with diabetic mellitus and cardiovascular diseases. Diabetes mellitus is characterized by hyperglycemia together with the biochemical alteration of glucose and lipid peroxidation [17-19]. Lipid peroxidation, a free radical related process, is an uncontrolled, self-enhancing process causing disruption of membrane lipid and other cell components. Unlimited lipid peroxidation (LP) could be one of the main factors in the pathogenesis of diabetic complications $[20,21]$. This pathology is often related to the release of free radicals and caused oxidative stress [22]. During re oxygenation hypoxanthine/ xanthine oxidase and arachidonic acid pathways are important sources of free oxygen radicals, which damage lipid membrane and lead to cytolysis and cell death [23].

The accumulation of lipid in diabetes is mediated through a variety of derangement in metabolic and regulatory process especially insulin deficiency thereby rendering the diabetic patient more prone to hypercholesterolemia and hypertriglyceridemia [24]. One of the major pathogenesis of lipid metabolism, disturbances in diabetes, is the increase mobilization of fatty acid from adipose tissue and secondary elevation of free fatty acid level in blood [25]. Lipid abnormalities such as hypercholesterolemia, hypertriglyceridemia, hyperphospholipidemia and fatty acid distribution changes are common in diabetic patients [14]. Consumption of soft drinks in Nigeria is becoming high, and this soft drinks are reported to contained high calories which tend to increase the risk of obesity, type-2 diabetes and cardiovascular diseases [26]. Therefore, there is need to study the effects of these soft drinks through the assessment of these parameters as indices that point to predisposure to diabetes mellitus and cardiovascular diseases in order to reduce the risk of obesity, type- 2 diabetes and cardiovascular diseases.

\section{Materials and Methods}

Collection of soft drinks

Coca-Cola soft drinks (Coke, Zero Coke, Fanta and Sprite) were purchased from Final Solution Catering Service shop at Odo-Ona, along Apata road, Ibadan, Nigeria and were kept at room temperature.

\section{Experimental design}

Twenty-five (25) adult Wistar rats (Rattus norvegicus) with body weight between 120 and $150 \mathrm{~g}$ were used for this study. They were acclimatized for seven (7) days during which they were fed ad libitum with standard feed and drinking water and were housed in clean cages placed in well-ventilated housing conditions (under humid tropical conditions) throughout the experiment. All the animals received humane care according to the criteria outlined in the 'Guide for the Care and Use of Laboratory Animals' prepared by the National Academy of Science and published by the National Institute of Health. They were randomly divided into 5 groups of 5 rats each and were treated according: animals in group 1 were administered distilled water, those in group 2 were administered Coke, those in group 3 were administered Zero Coke, those in group 4 were administered Fanta, while those in group 5 were administered Sprite. The administration was done orally at a dose of $3 \mathrm{~mL}$ per $100 \mathrm{~g}$ body weight 12 hourly for fourteen days. At the end of administration period, the animals were fasted overnight and anaesthetized using diethyl ether. Blood samples were collected by cardiac puncture.

\section{Determination of fasting blood sugar}

Fasting blood sugar was determined according to the methods described by Airaodion., et al. [27]. After the acclimatization period, animals used in this study were allowed to fast for twelve (12) hours before the commencement of administration. The blood glucose level were taken by sterilizing the tails of the animals with $10 \%$ alcohol, and cutting the tails using scissors then allowing the blood to touch the test strip which was inserted into a calibrated glucose meter (One touch Glucometer, Acon Laboratory INC. San Diego, USA). This gave direct reading after 5 seconds in $\mathrm{mg} / \mathrm{dL}$. The blood glucose level of the rats before the commencement of administration was measured in order to know the normal blood glucose of the rats in each group. At the end of administration, all the rats in the each group were fasted overnight and their fasting blood sugar was determined using glucose meter. This was done in order to check and observe the effect of soft drinks on blood glucose level when compared to their initial glucose level (before the administration).

\section{Determination of lipids}

Lipids were extracted and determined according to previously described methods [28,29].

\section{Statistical analysis}

Data were subjected to analysis of variance using Graph Pad Prism. Results were presented as Mean \pm standard deviation. One way analysis of variance (ANOVA) was used for comparison of the means followed by Tukey's (HSD) multiple comparison tests. Differences between means were considered to be significant at $\mathrm{p}<0.05$.

\section{Results}

The results of the effect of Coca-Cola soft drinks on fasting blood glucose and lipid profile of Wistar rats are presented in tables 1 and 2 respectively. 


\begin{tabular}{|l|c|c|c|}
\hline \multicolumn{1}{|c|}{ Treatment } & Pre-treatment (mg/dL) & Post-treatment (mg/dL) & Increase in FBG (mg/dL) \\
\hline Control & 58.824 .32 & 60.073 .94 & $1.250 .02^{\mathrm{a}}$ \\
\hline Coke & 60.352 .03 & 98.197 .02 & $37.843 .24^{\mathrm{b}}$ \\
\hline Zero Coke & 61.253 .28 & 64.113 .89 & $2.860 .45^{\mathrm{a}}$ \\
\hline Fanta & 59.226 .09 & 93.456 .62 & $34.235 .12^{\mathrm{b}}$ \\
\hline Sprite & 60.424 .34 & 72.003 .48 & $11.582 .44^{\mathrm{c}}$ \\
\hline
\end{tabular}

Table 1: Effect of Coca-Cola Soft Drinks on the Fasting Blood Glucose (FBG) of Animals after 14 days Administration.

Values are presented as MeanS.E.M, where $\mathrm{n}=5$. Values with different superscript along the same column are significantly different at $\mathrm{p}<0.05$.

\begin{tabular}{|l|c|c|c|c|c|c|}
\hline Treatment & TC (mg/dL) & TG (mg/dL) & HDL (mg/dL) & LDL (mg/dL) & HDL:LDL & VLDL (mg/dL) \\
\hline Control & $98.258 .29^{\mathrm{a}}$ & $88.487 .43^{\mathrm{a}}$ & $44.836 .49^{\mathrm{a}}$ & $41.643 .92^{\mathrm{a}}$ & $1.080 .02^{\mathrm{a}}$ & $17.701 .48^{\mathrm{a}}$ \\
\hline Coke & $126.1111 .16^{\mathbf{b}}$ & $122.0314 .39^{\mathrm{b}}$ & $34.923 .29^{\mathrm{b}}$ & $51.245 .25^{\mathrm{b}}$ & $0.680 .02^{\mathrm{b}}$ & $24.412 .23^{\mathrm{b}}$ \\
\hline Zero Coke & $99.045 .45^{\mathrm{a}}$ & $90.054 .62^{\mathrm{a}}$ & $41.373 .84^{\mathrm{a}}$ & $42.047 .73^{\mathrm{a}}$ & $0.980 .12^{\mathrm{a}}$ & $18.011 .53^{\mathrm{a}}$ \\
\hline Fanta & $128.639 .27^{\mathrm{b}}$ & $113.338 .56^{\mathbf{c}}$ & $32.664 .04^{\mathrm{b}}$ & $49.924 .94^{\mathrm{a}}$ & $0.650 .10^{\mathrm{b}}$ & $26.673 .41^{\mathrm{b}}$ \\
\hline Spirit & $101.526 .77^{\mathrm{a}}$ & $97.643 .54^{\mathrm{d}}$ & $40.525 .72^{\mathrm{a}}$ & $40.223 .27^{\mathrm{b}}$ & $1.010 .15^{\mathrm{b}}$ & $19.532 .93^{\mathrm{a}}$ \\
\hline
\end{tabular}

Table 2: Effect of Coca-Cola Soft Drinks on the Lipid Profile of Animals after 14 days Administration. Values are presented as MeanS.E.M, where $n=5$. Values with different superscript along the same row are significantly different at $\mathrm{p}<0.05$.

Legend: TC: Total Cholesterol; TG: Triglyceride; HDL: High Density Lipoprotein;

LDL: Low Density Lipoprotein; VLDL: Very Low Density Lipoprotein.

\section{Discussion}

Diabetes is a complex metabolic disorder associated with developing insulin resistance, impaired insulin signaling and $\beta$-cell dysfunction, abnormal glucose and lipid metabolism, sub-clinical inflammation and increased oxidative stress. These metabolic disorders lead to long-term pathogenic conditions including micro- and macro-vascular complications, neuropathy, retinopathy, nephropathy, and a consequent decrease in quality of life and an increase in the rate of mortality [30]. Among the multiple risk factors underlining the incidence and progression of diabetes, diet is the main modifiable factor. Both experimental and epidemiological evidences have shown that consumption of vegetables rich in phenolic compounds and possess high antioxidant capacity may have inverse relationship with the incidence and prevalence of diabetes [31]. Dietary control remains one of the most desirable avenues for the prevention and management of chronic degenerative diseases such as diabetes and cardiovascular diseases. This study sought to investigate the effect of oral intake of Coca-Cola soft drinks in fasting blood glucose and lipid profile of Wistar rats.

In this study, all the Coca-Cola soft drinks (except zero coke) were observed to significantly elevated the fasting blood glucose of animals at $\mathrm{p}<0.05$. This might be an indication that these drinks could predispose consumers to the risk of diabetes. One therapeutic approach for treating early stage of diabetes is to decrease postprandial hyperglycaemia. This is done by retarding the absorption of glucose through the inhibition of the carbohydrate-hydrolyzing enzymes, $\alpha$-amylase and $\alpha$-glucosidase, in the digestive tract. Consequently, activators of these enzymes determine an elevation in the rate of glucose absorption and consequently blunting the post- prandial plasma glucose rise [32,33]. Based on these findings, it could be suggested that these soft drinks may stimulate platelet aggregation and reduce vasodilatation, exerting an important role in the onset, development and progression of vascular complications caused by the hyperglycemic state [34]. However, zero coke had no significant effect on the fasting blood glucose of animals when compared with the control group after fourteen days treatment. This is suggestive that of all the Coca-Cola soft drinks used in this study, only zero coke is safe for diabetic patients and those predisposed to diabetes to consume. This result corresponds to the findings of Airaodion., et al. [35] who treated animals with Bigi soft drinks. The fasting blood glucose of animals treated with sprite in this study was significantly higher than those of control animals but significantly lower than those in animals treated with Coke and Fanta at $\mathrm{p}<0.05$ respectively (table 1 ). This contradicts the findings of Goje., et al. [36] who reported a nonsignificant difference in the fasting blood glucose of animals fed with sprite when compared with those of control animals.

Apart from the regulation of carbohydrate metabolism, insulin plays an important role in lipid metabolism. Insulin insufficiency, as in diabetes mellitus, is associated with hypercholesterolemia and hypertriglyceridemia, which have been reported to occur in experimental diabetic rats [37-39]. Hypercholesterolemia could result in a relative molecular ordering of the residual phospholipids, resulting in a decrease in membrane fluidity [40]. Accumulation of triglycerides is one of the leading risk factors in coronary heart disease (CHD) [35]. Lipid and lipoprotein abnormalities have been shown to play a major role in the pathogenesis and progression of several disease conditions [41]. 
In this study, total cholesterol concentrations were observed to increase significantly when animals treated with Coke and Fanta were compared with those of the control group at $\mathrm{p}<0.05$ (Table 2 ). This could be that these soft drinks may increase the progression of CHD. However, Zero Coke and Sprite has no significant effect on the total cholesterol of animals after fourteen days treatment. This corresponds with the findings of Goje., et al. [36] who reported a nonsignificant difference in the total cholesterol of animals fed with sprite when compared with those of control animals.

Significant increase was observed in the triglyceride levels of all animals treated with soft drinks except those treated with Zero Coke whose triglyceride level was not significantly different from those of control animals. Hypertriglyceridaemia has been reported in diabetic animals [42]. This was reported to be due to increased absorption and formation of triglycerides in the form of chylomicrons following exogenous consumption of diet rich in fat or through increased endogenous production of triglycerideenriched hepatic VLDL-cholesterol and decreased triglyceride uptake in peripheral tissues [42]. Hypercholesterolaemia has also been reported in diabetic animals [42]. This was attributed to the increased dietary cholesterol absorption from the small intestine following the intake of high fat diet in a diabetic condition [43]. Moreover, it can be conjectured that the lipid increasing effects of Coca-Cola soft drinks could be due to the stimulation of hepatic cholesterol, triglyceride and possibly fatty acid synthesis [44]. The elevation in the triglyceride and total cholesterol observed in this study might be an indication that these soft drinks has the propensity to induce hypertriglyceridaemia and hypercholesterolaemia respectively making them to be potent in the induction of diabetes mellitus and cardiovascular diseases. The nonsignificant different in the concentrations of total cholesterol and triglyceride observed in animals treated with Zero Coke might indicate that while other Coca-Cola soft drinks used in this study may induce hypertriglyceridaemia and hypercholesterolaemia, Zero Coke might not.

The triglyceride level of animals treated with sprite in this study was significantly higher than those of control animals but significantly lower than those of animals treated with Coke and Fanta at $\mathrm{p}<0.05$ respectively (table 2 ). This contradicts the findings of Goje., et al. [36] who reported a nonsignificant difference in the triglyceride concentration of animals fed with sprite when compared with those of control animals. Hypertriglyceridaemia has also been reported to be a predictor of hypertension risk [45]. In the peripheral vascular system, endothelial cells rely on lipoproteins for the transfer of neutral sterols at this site. Although free cholesterol is transferred to HDL-cholesterol particles through the functioning of a designated HDL-cholesterol receptor, lecithin cholesterol acyl transferase (LCAT) serves to maintain the concentration toward the HDL core and preserve the hydrophobic nature that facilitates the transfer. Esterification of cholesterol produces cholesterol ester (CE), which is concentrated in HDL core, and may be transferred by cholesterol ester transfer protein (CETP) in the plasma compartment to apo-B containing lipoproteins in exchange for triglyceride. Increased CETP activity would suggest an enrichment of apo-B lipoproteins in plasma, while simultaneously decreasing HDL-cholesterol, and has generally been considered proatherogenic [46]. This probably explains why Coca-Cola soft drinks may lead to an elevation in the risk of developing heart diseases since a low HDL-cholesterol/LDL-cholesterol ratio has been shown to be deleterious and is indicative of a higher risk of cardiovascular diseases [47].

HDL-cholesterol and LDL-cholesterol are two of the four main groups of plasma lipoproteins that are involved in lipid metabolism and the exchange of cholesterol, cholesterol ester and triglycerides between tissues $[48,49]$. Numerous population studies have shown an inverse correlation between plasma HDL-cholesterol levels and risk of cardiovascular disease, implying that factors associated with HDL-cholesterol protect against atherosclerosis. Some of these factors appear to have antioxidant and anti-inflammatory effects which may obviate processes that initiate atherogenesis $[50,51]$.

Epidemiological studies have also shown that elevated concentrations of total cholesterol and/or LDL-cholesterol in the blood are powerful risk factors for coronary heart disease [35,52]. Most extra-hepatic tissues, although having a requirement for cholesterol, have low activity of the cholesterol biosynthetic pathway. Their cholesterol requirements are supplied by LDL, which is internalized by receptor-mediated endocytosis. A major function of HDL-cholesterol is to enhance reverse cholesterol transport by scavenging excess cholesterol from peripheral tissues followed by esterification through lecithin: cholesterol acyltransferase and delivering it to the liver and steroidogenic organs for subsequent synthesis of bile acids and lipoproteins and eventual elimination from the body $[53,54]$. This role of HDL-cholesterol has been shown to be responsible for its atheroprotective properties. HDL-cholesterol also regulates the exchange of proteins and lipids between various lipoproteins.

In addition, HDL-cholesterol provides the protein components required to activate lipoprotein lipase which releases fatty acids that can be oxidized by the ß-oxidation pathway to release energy $[35,49]$. Most importantly, HDL-cholesterol can inhibit oxidation of LDL-cholesterol as well as the atherogenic effects of oxidized LDLcholesterol by virtue of its antioxidant property [45]. LDL is a lipoprotein that transports cholesterol and triglyceride from the liver to peripheral tissues. It enables fat and cholesterol to move within the water-blood solution of the blood stream. LDL is often called bad cholesterol; hence low levels are beneficial [55].

Unfortunately, the administration of Coke and Fanta in this study caused a significant decrease in the serum level of HDL-cholesterol when compared with the control animals at $\mathrm{p}<0.05$ (table 2). This contradicts the findings of Goje., et al. [36] who reported a nonsignificant difference in the HDL-cholesterol of animals treated with Fanta when compared with those in animals in the control group. HDL-cholesterol is usually referred to as the 'good cholesterol' [35]. Again, administration of Coke and Fanta significantly 
increased the concentration of LDL-cholesterol (bad cholesterol) when compared with that of the control group at $\mathrm{p}<0.05$. This also contradicts the findings of Goje., et al. [36] who reported a nonsignificant difference in the LDL-cholesterol of animals treated with Coke when compared with those in animals in the control group. The combined effect of decreased HDL-cholesterol (good cholesterol) and increased LDL-cholesterol (bad cholesterol) in the present study resulted in a decreased HDL-cholesterol/LDL-cholesterol ratio in animals treated with Coke and Fanta when compared with the control group. This strongly supports the notion that dietary supplementation with the extract of soft drinks may lead to an elevation in the risk of developing heart diseases, because a low HDL-cholesterol/LDL-cholesterol ratio has been shown to be deleterious and is indicative of a higher risk of CHD [56]. Although, the activities of enzymes were not investigated in this study, but it is possible that Coca-Cola soft drinks increased the activity of 3-hydroxy-3-methylglutaryl coenzyme A (HMG CoA) reductase (the rate-limiting enzyme in cholesterol biosynthesis) [29,35]. This implies that consumption of some Coca-Cola soft drinks are of adverse health importance as far as hyperlipidemia is concern.

The result of this study showed that Sprit may be safe for people with cardiovascular diseases history and also cardiovascular disease patients but may predispose one to diabetes on long term consumption. However, Zero Coke with no significant effect on the fasting blood glucose and lipid profile of animals may the safe for consumption as far as hyperglycemia and hyperlipidemia is concern.

\section{Conclusion}

The result of this study implies that Coke and Fanta might be deleterious to health as far as hyperglycemia and hyperlipidemia is concerned. Individuals with diabetic family history should minimize their consumption of these drinks but rather consume Zero Coke.

\section{Conflict of Interest}

Authors declare that no conflict of interest exist in this paper.

\section{Bibliography}

1. Vartanian LR., et al. "Effects of soft drink consumption on nutrition and health: a systematic review and meta-analysis". American Journal of Public Health 97 (2007): 667-675.

2. Ebbeling CB., et al. "Effects of decreasing sugar-sweetened beverage consumption on body weight in adolescents: A randomized, controlled pilot study". Pediatrics 117 (2006): 673680 .

3. Malik VS., et al. "Intake of sugar sweetened beverages and weight gain: a systematic review". American Journal of Clinical Nutrition 84 (2006): 274-328.

4. Wolff E and Dangsinger ML. "Soft drinks and weight gain: How strong is the link?". Medscape Journal of Medicine 10 (2008): 189.
5. Caroline DW. "Type 1 diabetes mellitus in pediatrics”. Pediatric Review 29 (2008): 374-384.

6. Gibson S. "Sugar-sweetened soft drinks and obesity: a systematic review of the evidence from observational studies and interventions". Nutrition Research Reviews 21 (2008): 134-147.

7. Ludwig D., et al. "Relation between consumption of sugarsweetened drinks and childhood obesity: a prospective, observational analysis". The Lancet 357 (2001): 505-508.

8. Schulze MB., et al. "Sugar sweetened beverages, weight gain, and incidence of type 2 diabetes in young and middle aged women". Journal of the American Medical Association 292 (2004): 927-934.

9. Dubois HFW and Bankauskaite V. "Type 2 diabetes programmes in Europe". Euro Observer 7 (2005): 5-6.

10. Ashurst P. "Soft drink and fruit juice problems solved". Woodhead Publishing Limited (2009).

11. Raben A., et al. "Sucrose compared with artificial sweeteners: different effects on ad libitum food intake and body weight after 10 weeks of supplementation in overweight subjects". American Journal of Clinical Nutrition 76 (2002): 721-729.

12. Nielsen $S$ and Popkin B. "Changes in beverage intake between 1977 and 2001". American Journal of Preventive Medicine 27 (2009): 205-210.

13. Ripsin CM., et al. "Management of blood glucose in type 2 diabetes mellitus". American family physician 79 (2009): 29-36.

14. Konstantinos L. "Aretaeus of Cappadocia and the first description of diabetes". Hormones 11 (2012): 109-113.

15. Ripoll BC and Leutholtz I. Exercise and disease management (2nd ed.). Boca Raton: CRC Press (2011): 25.

16. Santaguida PL., et al. "Diagnosis, prognosis, and treatment of impaired glucose tolerance and impaired fasting glucose". Evidence Report Technology Assessment 12 (2008): 1-11.

17. Trocho $\mathrm{C}$ and Pardo RT. "Formaldehyde derived from dietary aspartame binds to tissue components in vivo". Life Science (1998) 637-349.

18. Lambert P and Bingley PJ. "What is Type 1 Diabetes?". Medicine 30 (2002): 1-5.

19. Rother KI. "Diabetes treatment-bridging the divide". The New England Journal of Medicine 356 (2007): 1499-1501.

20. Stewart WF., et al. "Lost productive time and costs due to diabetes and diabetic neuropathic pain in the US workforce". Journal of Occupational and Environmental Medicine 49 (2007): 672-679. 
21. Shoback DG and Gardner D, Eds. "Chapter 17". Greenspan's Basic and Clinical Endocrinology (9th Ed.). New York: McGraw-Hill Medical (2011).

22. Saydah SH., et al. "Postchallenge hyperglycemia and mortality in a national sample of U.S. adults". Diabetes Care 24 (2001): 1397-1402.

23. Dorner M., et al. "Essential labile diabetes" (in German). Munch Med Wochenschr 119 (1977): 671-674.

24. Jaiprakash J., et al. "Preventing childhood obesity by reducing consumption of carbonated drinks: cluster randomized controlled trial". British Medical Journal 328 (1993): 1237.

25. Vijan, S. "Type 2 diabetes". Annals of Internal Medicine 152 (2010): ITC31-15.

26. Selvin E., et al. "Glycated hemoglobin, diabetes, and cardiovascular risk in nondiabetic adults". The New England Journal of Medicine 362 (2010): 800-811.

27. Airaodion AI., et al. "Effect of Oral Intake of African Locust Bean on Fasting Blood Sugar and Lipid Profile of Albino Rats". Asian Journal of Research in Biochemistry 4 (2019): 1-9.

28. Owoade AO., et al. "Toxicological assessment of the methanolic leaf extract of Bridelia ferrugelia". The Journal of Phytopharmacology 7 (2018): 419-424.

29. Owoade AO., et al. "Levofloxacin-induced dyslipidemia in male albino rats". Asian Journal of Pharmacy and Pharmacology 4 (2018): 620-629.

30. Airaodion AI., et al. "Pharmacotherapeutic effect of methanolic extract of Telfairia occidentalis leaves on glycemic and lipidemic indexes of alloxan-induced diabetic rats". International Journal of Bio-Science and Bio-Technology 11 (2019): 1-17.

31. Bahadoran Z., et al. "The association of dietary phytochemical index and cardio-metabolic risk factors inadults: Tehran lipid and glucose study". Journal of Human Nutrition and Diet (2013).

32. Airaodion AI., et al. "Comparative assessment of phytochemical content and antioxidant potential of Azadirachta indica and Parquetina nigrescens leaves". Asian Plant Research Journal 2 (2019): 1-14.

33. Airaodion AI., et al. "Evaluation of Phytochemical Content and Antioxidant Potential of Ocimum gratissimum and Telfairia occidentalis Leaves". Asian Journal of Research in Medical and Pharmaceutical Sciences 7 (2019): 1-11.

34. Airaodion AI., et al. "Effect of methanolic extract of Corchorus olitorius Leaves on hypoglycemic and hypolipidaemic activities in albino rats". Asian Plant Research Journal 2 (2019): 1-13.
35. Airaodion AI., et al. "Bigi soft drinks might induce hyperglycemia and hyperlipidemia in Wistar rats'. International Journal of Research and Reports in Hematology 2 (2019): 1-10.

36. Goje CJ., et al. "Effect of the oral intake of some soft drinks on the fasting blood glucose level and lipid profile of albino rats". International Journal of Sciences 3 (2014): 70-75.

37. Loci AS., et al. "Hypoglycemic effect of a valuable extract on some blood parameters in diabetic animals". Journal of Ethnopharmacology 43 (1994): 167-171.

38. Ahardh CD., et al. "The effect of tolnetamide in lipoproteins and lipoprotein lipase and hormone sensitive lipase". Diabetes Research and Clinical Practice 46 (1999): 99-108.

39. Frayn KN. "Insulin resistance and lipid metabolism". Current Opinion on Lipidology 4 (1993): 197-204.

40. Bopanna KN., et al. "Antidiabetic and antihyperlipidemic effect of neem seed, kernel powder on alloxan diabetic rabbits". Indian Journal of Pharmacology 29 (1997): 162-167.

41. Rotimi OS., et al. "Amoxillinand pefloxacin-induced cholesterogenesis and phospholipidosis in rat tissues". Lipids in Health and Disease 201514 (2015): 13-30.

42. Airaodion AI., et al. "Antidiabetic Effect of Ethanolic Extract of Carica papaya Leaves in Alloxan-Induced Diabetic Rats". American Journal of Biomedical Science and Research 5 (2019): 227-234.

43. Srinivasan K., et al. "Combination of high-fat diet-fed and lowdose streptozotocin-treated rat: a model for type 2 diabetes and pharmacological screening". Pharmacological Research 52 (2005): 313-320.

44. Balamurugan R and Ignacimuthu S. "Antidiabetic and Hypolipidemic effect of methanol extract of Lippia nodifloraL. in streptozotocin induced diabetic rats". Asia Pacific Journal of Tropical Biomedicine (2011): S30-S36.

45. Allen RR., et al. "Daily consumption of a dark chocolate containing flavanols and added sterol esters affects cardiovascular risk factors in normotensive population with elevated cholesterol". Journal of Nutrition 138 (2008): 725-731.

46. Greene CM., et al. "Maintenance of the LDL-cholesterol/ HDLcholesterol ratio in an elderly population given a dietary cholesterol challenge". Journal of Nutrition 135 (2005): 27932798.

47. Perona JS., et al. "Reduction in systemic and VLDL triacylglycerol concentration after a 3-month Mediterranean style diet in high-cardiovascular-risk subjects". Journal of Nutrition and Biochemistry 9 (2010): 892-898. 
48. Gordon DJ and Rifkind BM. "High-density lipoprotein: The clinical implications of recent studies". New England Journal of Medicine 321 (1989): 1311-1316.

49. Sviridiv D. "Intracellular cholesterol trafficking". Histology and Histopathology 14 (1999): 305-319.

50. Airaodion AI., et al. "Hypoglycemic and hypolipidaemic activities of methanolic extract of Talinum triangulare leaves in Wistar rats". International Journal of Bio-Science and Bio-Technology 11 (2019): 1-13

51. Oram JF and Lawn RM. "ABCA1: The gatekeeper for eliminating excess tissue cholesterol". Journal Lipid Research 42 (2001): 1173-1179.

52. Law MR. "Lowering heart disease risk with cholesterol reduction: Evidence from observational studies and clinical trials". European Heart Journal 1 (1999): S3-S8.

53. Stein 0 and Stein Y. "Atheroprotective mechanisms of HDL". Atherosclerosis 144 (1999): 285-303.

54. Ogbuagu EO., et al. "Effect of methanolic extract of Vernonia amygdalina leaves on glycemic and lipidaemic indexes of Wistar rats". Asian Journal of Research in Medical and Pharmaceutical Sciences 7 (2019): 1-14.

55. Cromwell WC and Otvos JD. “Low Density Lipoprotein Particle Number and Risk for Cardiovascular Disease". Current Atherosclerosis Reports 6 (2004): 381-387.

56. Castelli L. "Epidemiology of coronary heart disease". American Journal of Medicine 76 (1984): 4-12.

Volume 3 Issue 12 December 2019

(C) All rights are reserved by Augustine I Airaodion. 